\section{Why the long latent period?}

\section{Charles R. M. Bangham and Andrew J. McMichael}

THE central problems in understanding the progression of infection with human immunodeficiency virus (HIV) to AIDS stem from the virus's long latent period: the median time from infection to development of the disease is about eight years. Two questions then arise. First, what is the role of the immune response in the progression to AIDS? More precisely, is the decline in the immune response the cause or consequence of the rise in HIV titre, and do antibodies or cytotoxic $\mathrm{T}$ lymphocytes (CTL) exert significant selection against HIV variant sequences? Second, what is the effect of the extreme sequence diversity of HIV: do variants arise that escape the immune response'? Nowak and colleagues, in a report published earlier this month in the journal $A I D S^{2}$, use a simple model to try to explain the long latent period on the basis of the sequence diversity of HIV. According to the model, AIDS is the result of an increase in the number of immunologically distinct variants of HIV above a critical threshold. How can this hypothesis be tested?

RNA viruses, such as HIV, are highly variable in sequence ${ }^{3}$, largely because of the absence of a 'proof-reading' 3 ' -5 ' exonuclease activity in the RNA-dependent polymerase. Variant sequences of an RNA virus arise frequently both in vitro and in vivo; the diversity within one infection, particularly a long-term persistent infection, may be greater than the diversity between infectious isolates, because many of the variants produced are defective in replication or infectivity, and so are not propagated. The mixed population of variant sequences of a virus has been called a 'quasispecies".

The main assumptions made in the new model are these: (1) HIV can kill any $\mathrm{CD}^{+}$cell, but only a subset of $\mathrm{CD}^{+}$cells are involved in killing a given variant of HIV; (2) antigenic variants of HIV arise which escape destruction by the immune system. The basic equation in the model is then $\mathrm{d} V_{\mathrm{i}} / \mathrm{d} t=V_{\mathrm{i}}\left(r-s z-p x_{\mathrm{i}}\right)$ where $V_{\mathrm{i}}$ is the population size of the variant sequence $\mathrm{i} ; t$ is time; $r$ is the net replication rate of the virus (initially assumed to be the same for each strain); $z$ is the number of 'immune agents' (antibodies or T cells) that recognize conserved epitopes; $x_{\mathrm{i}}$ is the number of immune agents specific to the variant sequence $\mathrm{i}$; and $s$ and $p$ are rate constants respectively for the effect of the cross-reactive and specific elements of the immune response.

On this model, the immune system can be said to control the variant i if $\mathrm{d} V_{\mathrm{i}} / \mathrm{d} t<0$, that is if $r-s z-p x_{\mathrm{i}}<0$. Summing over all $n$ variant sequences of the virus, we have $n(r-s z)-p \sum x_{\mathrm{i}}<0$, that is $n<p \sum x_{\mathrm{i}} /(r-s z)$. The authors show that when the number $n$ of HIV variants exceeds a critical value $n_{\mathrm{c}}=p \sum x_{\mathrm{i}} /(r-s z)$, the total virus load increases steadily with time.

The model includes a stochastic term which simulates the emergence of variants that are initially not neutralized by the pre-existing immune response. As the immune system responds, each successive variant is temporarily controlled, until the number of variants exceeds the threshold

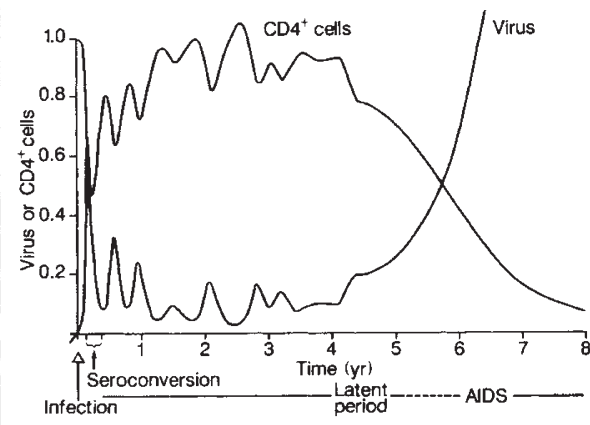

Changes in the numbers of $\mathrm{CD} 4^{+}$cells and HIV titre (arbitrary scale), following infection with HIV, predicted by the model proposed by Nowak et al. ${ }^{2}$.

value $n_{\mathrm{c}}$, after which all the variants can multiply unhindered and kill the remaining $\mathrm{CD}^{+}$cells

The authors then show that a more realistic model, including notably the assumption that different HIV variants replicate at different rates, does not qualitatively alter the outcome. The resulting changes in the $\mathrm{CD} 4{ }^{+}$cell number and HIV titre occurring over the years during progression to AIDS are summarized in the figure, and they correspond well to what has been observed in humans ${ }^{5}$.

Attempts to explain the initial control of HIV and the eventual breakdown of the immune system in terms of simple immunological mechanisms are fraught with danger because the population dynamics of cells and virus rapidly become complex, and this complexity is increased greatly by the sequence diversity of the virus. The usefulness of models such as that proposed by Nowak et al. is to identify what seem to be the important factors determining disease progression. The clear implication of the model is that the most important single factor leading to the breakdown of immune control of HIV is the increase in the diversity of the virus. If this is correct, we should not be examining the predominant HIV sequence(s) isolated during the symptomatic phase of HIV infection to look for 'determinants of virulence'. This variant may simply be a fast-replicating one that was around when the immune system broke down.

However, tests of the hypothesis will have to be formulated with great care. The principal difficulty is that what we need to measure is not the sequence diversity but the antigenic diversity of HIV: the assays should therefore be immunological, and should not rely simply on sequence comparisons. Further, detection of variant HIV sequences depends mostly on the use of the polymerase chain reaction (PCR), but this technique fails to distinguish between functional and defective variants of HIV. We know that a high proportion of integrated proviruses are defective, 1015 per cent in tat alone 6 . Presumably we should therefore try to identify replication-competent variants by growing them in cell culture: the snag there is that HIV continues to undergo mutation in vitro ${ }^{6}$. These problems are magnified in the silent phase of HIV infection, during which the viral abundance is low: it is therefore still more difficult to get an accurate measure of the number of variant sequences, and to culture the virus for long periods is to select for fast-replicating variants.

A logical approach is perhaps as follows: prepare complementary DNA from the peripheral blood lymphocytes of an HIVinfected person either directly or after a brief period (say, up to one week) in culture, so that replication-competent strains begin to grow but do not diversify further (and one fast-replicator does not predominate); then amplify by PCR the regions of the HIV genomes that are known to encode important CTL, or antibody epitopes, such as the V3 loop of Env (antibody epitope) or the Gag (CTL epitopes) proteins. There is variation in the sequence of Gag CTL epitopes, even in highly conserved regions of gag (ref. 7; R. E. Phillips, personal communication). This approach involves not only sequence determination, but both antibody and CTL assays to test the antigenic distinctness of a new variant. And it depends on a knowledge of the most important epitopes in subjects of known HLA genotype.

A potentially important corollary of the model, noted by the authors, is that any agent such as a drug which reduces the replication rate of the virus should increase the critical number of HIV strains required to cause AIDS, and so reduce the chance of disease progression. Is this how AZT works?

Charles R. M. Bangham and Andrew J. McMichael are in the Institute for Molecular Medicine, University of Oxford, John Radcliffe Hospital, Oxford OX3 9DU, UK.

1. Pircher, H. et al. Nature 346, 629-633 (1990).

2. Nowak, M. A., May, R. M. \& Anderson, R. M. AIDS4, 1095 $1.103(1990)$

Steinhauer, D. A. \& Holland, J. J. A. Rev. Microbiol. 41 409-433 (1986)

4. Eigen, M. Naturwissenschaften $\mathbf{5 8}, 465-523$ (1971).

5. Miedema, F., Tersmette, M. \& van Lier, R. A. W. Immun Today 11, 293-297 (1990)

6. Meyerhans, A. et al. Cell 58, 901-910 (1989).

7. Goodenow, M. et al. J. Acq. Immun. Def. Syn. 2, 344-352 (1989). 